\title{
Modeling of conductive heat transfer in a 3D numerical material by a stochastic process
}

\author{
V. Gonneau ${ }^{1}$, D. Rochais ${ }^{1}$, F. Enguehard ${ }^{2}$, S. Chupin ${ }^{1}$, G. L. Vignoles ${ }^{3}$ \\ ${ }^{1}$ CEA DAM Le Ripault \\ vincent.gonneau@cea.fr \\ ${ }^{2}$ Institut Prime, CNRS, Université de Poitiers, ISAE-ENSMA \\ F-86962 Futuroscope Chasseneuil, France \\ ${ }^{3}$ LCTS, UMR 5801 CNRS
}

\section{Extended Abstract}

In many high temperature applications, porous refractory materials (for example alumina $\mathrm{Al}_{2} \mathrm{O}_{3}$ or zirconia $\mathrm{ZrO}_{2}$ felts) have become serious candidates as insulating materials. However, the modeling of heat transfer in these porous materials requires to solve several difficulties. First of all, the complex morphology of the medium makes it very difficult to realize a mesh for finite element or finite volume methods. Moreover, these methods require high amounts of computer storage and/or are time consuming. Thus, a 3D numerical voxel structure, issued from X-ray tomography for example, has been chosen to represent the material, allowing to avoid the meshing process. A second difficulty is the resolution of transient conduction transfer in these porous materials and the treatment of the boundary conditions in a numerical structure.

The aim of this study is to develop a new modeling procedure of transient conduction in a numerical felt. The transient thermal conduction transfer is described as a stochastic process based on the motion of brownian walkers, a modeling approach that was initiated in [1]. Every walker carries an elementary enthalpy and the temperature of a volume $\mathrm{V}$ is proportional to the number of walkers within it. A computer code based on brownian walker motion was developed. Different boundary conditions were studied and implemented such as adiabatic wall, imposed thermal flux or imposed temperature. The code ensures the physical continuity of heat flux at interfaces and is able to model internal sources within the porous material. This study presents methods to set up boundary conditions and interface processing.

Our stochastic treatment of transient thermal conduction was validated, in 1D transfer configurations, by comparison with Comsol results. Moreover, CEA has already developed a transient heat transfer numerical model operating on 3D voxel structures based on the deterministic finite difference method [2]. The stochastic (based on Brownian motion) and deterministic (based on finite differences) numerical tools are applied to a 3D numerical structure issued from X-ray tomography. This study compares the simulated temperature fields in this structure obtained by these two different modeling approaches, and highlights the strengths and weaknesses of each approach. In the future, our brownian motion based transient conduction model will be coupled to radiation heat transfer by the injection of a radiative heat source term. This radiative source will be determined by the resolution of the radiative transfer equation.

\section{References}

[1] G. L. Vignoles, "A hybrid random walk method for the simulation of coupled conduction and linearized transfer at local scale in porous media with opaque solid phases," International Journal of Heat and Mass Transfer, vol. 93, pp. 707719, 2016.

[2] M. Niezgoda, D. Rochais, F. Enguehard, B. Rousseau, P. Echegut, "Modeling heat transfer within porous multiconstituent materials," Journal of Physics: Conference Series, vol. 369, 2012. 